\title{
Intelligent Line Map Robot Using Matlab
}

\author{
Mr. Prashant Maruti Jadhav ${ }^{1}$, Mrs. Poonam Babasaheb Bhopale ${ }^{2}$ \\ Assistant Professor Electrical Engineering Department DKTE Society's TEI, Ichalkaranji, India ${ }^{1}$ \\ Assistant Professor ETC Department DKTE’S Yashvantrao Chavan Polytechnic, Ichalkaranji, India ${ }^{2}$
}

\begin{abstract}
Robotics has become a very important application in most of the developed areas. High performance, high accuracy, lower labor cost and the ability to work in hazardous places have taken robotics in an advantages position over many other such technologies. Robots produce more accurate and high-quality work. Robots rarely make mistakes and are more precise than human workers. They can produce a greater quantity in a short amount of time. They can work at a constant speed with no breaks, days off, or holiday time. They can perform applications with more repeatability than humans. Robots save workers from performing dangerous tasks. They can work in hazardous conditions, such as poor lighting, toxic chemicals, or tight spaces. They are capable of lifting heavy loads without injury or tiring. Robots increase worker safety by preventing accidents since humans are not performing risky jobs. Work cells provide safety features, separating the worker from harm's way. We saw the robots like wired robot, Wireless robot, Path follower robot, Color detection robot where the user is always engaged with the robot. So, to overcome from such problem's GUI based robot is introduced in real time application. Matlab can be considered as a CPU platform that can follow a map. The basic objective of Matlab is to provide cost effective solutions for industrial applications, commercial applications and security applications, but not restricted to them. GUI modified version of Matlab may be creatively used even for home applications too. Here we have selected some sample scenarios to highlight the useful occasions.
\end{abstract}

Keywords: Intelligent Robot, Matlab, Arduino, Map follower.

\section{INTRODUCTION}

In today's life, robots are most popular in automation industries. When age of automation started, machines began to take over the jobs, which were previously performed by humans. As time changes, now-days robots are most of tasks are performed by automation. So, in automation industries robot plays a vital role. Some of the examples of automation are$\mathrm{NC}$ and $\mathrm{CNC}$ machines, Feedback control machines, Automatic bottle filling machine, Automatic weather broadcasting, Process control in different industrial plants, Automatic assembly line etc. Robot is one of the examples of Automation.

There are mainly two types of Automations-

- Hard Automation

- Soft Automation

In hard automation for developing new products (means change in previous product like its size, shape, weight, quality etc), we required to change hardware. Capital cost required for hard automation is more. By changing in software/program if products size, shape, volume, weight etc changes then it considered as soft automation. Mechanical setup for soft automation is constant only software/ programming is changed.

If we highlight advantages of automation then we want to consider regarding its productivity i.e. high, better quality, efficient use of raw material, worker safety and this list is going on. The first industrial robot was introduced in U.S. in the 1960s. Since then, their technology has improved immensely creating many advantages of robots. The advancement of robotics has also caused robots to become more widespread across various industries ranging from manufacturing to health care. Many benefits of robots seem to be most noticeable in productivity, safety, and in saving time and money. Robot applications are widely spread throughout the world.

Robotics has become a very important application in most of the developed areas. High performance, high accuracy, lower labor cost and the ability to work in hazardous places have put robotics in an advantages position over many other such technologies. The robots can perform the tasks which the humans find them dangerous, boring or difficult, They can do the work with constant speed and precision and they continue and finish the work without feeling sick. The robots can be programmed to perform a simple task, they repeat that task more times, the robots work in the factory with high degree of accuracy and they work with constant velocity.

The robots help increase the number of manufactured products and decrease the production of defective goods, they can produce the same quality products during the production process, They do not get exhausted and they work for a long 


\section{DOI $10.17148 /$ IJIREEICE.2020.8801}

period of time .The robots can be used in the computer industry, they are used in all kinds of electronics from the radios to the microwaves, they are used in packaging, they are used in producing the food, textiles and the drugs. We saw the robots like wired robot, Wireless robot, Path follower robot, Color detection robot where the user is always engaged with the robot. So to overcome from such problems GUI based robot is introduced in real time application. Matlab can be considered as a CPU platform that can follow a map. The basic objective of Matlab is to provide cost effective solutions for industrial applications, commercial applications and security applications, but not restricted to them. GUI modified version of Matlab may be creatively used even for home applications too. Here we have selected some sample scenarios to highlight the useful occasions.

\section{A. MOTIVATION}

Robotics has become a very important application in most of the developed areas. High performance, high accuracy, lower labor cost and the ability to work in hazardous places have take robotics in an advantages position over many other such technologies. In history we saw the robots like wired robot, Wireless robot, Path follower robot, Color detection robot where the user is always engaged with the robot. So to overcome from such problems GUI based robot is introduced in real time application. In industrial sectors the goods carrier are either manually operating vehicles or path follower Robot but there are some drawbacks in this two methods. The human is continuously engaged with the vehicle and also chances of human mistakes. The second one is path following Robot which only follows the path. If you want to change the structure of Industry so at that time it will make problem. So we want to design Robot who survive in Dynamic condition, perform reliably and efficiently also user convenient. So by using GUI in Matlab we design User Convenient Map Follower Robot.

\section{LITERATURE REVIEW}

\section{LINE FOLLOWER ROBOT}

The line follower robot has great importance in industrial manufacturing process, automation, carrying cartage in a specific direction etc. Importance is given in this paper in investigating efficiency of the robot, response of the sensor, getting actual data of the sensors, feedback of the central processing unit depending on this response, error correction of following line, future aspects of the line follower robot, providing some real time data of the robot and giving the preliminary steps on fabricating a line follower robot. This robot is the basic form of the line follower robots. Much more complex form of line following robot can be manufactured depending on this basic form of line follower robot. More specifically, efforts has been put on acquiring data during test runs so that robots can be manufactured in massive way under specific requirements of purpose.

\section{WIRELESS COLOR SENSING ARM ROBOT}

This paper presents the implementation of wireless arm robot using Programmable System on Chip (PSoC) and virtual Instruments programming using Lab-VIEW graphical language. The wireless control has been implemented with Zigbee protocol. Some of the main features of Zigbee protocol are to establish wireless communication medium between the arm robot and the PC controller. The hardware system is based on the PSoC microcontroller interfaced wirelessly with LabVIEW via a Zigbee module. The user can send the control instructions to the robot from the PC by using the Zigbee Protocol. As soon as the instruction is received by another Zigbee at the receiver end, PSoC microcontroller will come into action and will start the motor drivers so that the Robot starts moving as per the instructions sent by the user/ operator from PC. In addition to the above qualities of the arm robot, its intelligence is further upgraded in this design by making use of a color sensor to identify and handle the required color objects.

\section{MATLAB-BASED SIMULATORS FOR MOBILE ROBOT SIMULTANEOUS LOCALIZATION AND MAPPING}

Mobile robot Simultaneous Localization and Mapping (SLAM) problem is one of the most active research areas in robotics. In the research and simulation of SLAM, MATLAB-based simulators are widely used due to their comprehensive functionalities and simple usage. In this paper, the main open source MATLAB-based simulators for SLAM and their properties are listed. Two representative ones are concretely introduced from the aspects of data creation and import, motion model and observation model, and algorithms implementation. Simulation results of these two simulators indicate that MATLAB-based simulators are convenient and helpful in the robot SLAM research when developing new algorithms and when comparing accuracy, consistency or convergence of different algorithms. The SLAM algorithms widely used in MATLAB-based simulators, including Extended Kalman Filter (EKF), Unscented Kalman Filter (UKF) based SLAM algorithm and Fast SLAM algorithm, are also introduced.

\section{PATH PLANNING OF AUTONOMOUS MOBILE ROBOT: A NEW APPROACH}

In this present work, we present an algorithm for path planning to a target for mobile robot in unknown environment. The proposed algorithm allows a mobile robot to navigate through static obstacles and finding the path in order to reach the target without collision. This algorithm provides the robot the possibility to move from the initial position to the final 


\section{DOI 10.17148/IJIREEICE.2020.8801}

position (target). The proposed path finding strategy is designed in a grid-map form of an unknown environment with static unknown obstacles. The robot moves within the unknown environment by sensing and avoiding the obstacles coming across its way towards the target. When the mission is executed, it is necessary to plan an optimal or feasible path for itself avoiding obstructions in its way and minimizing a cost such as time, energy and distance. The proposed path planning must make the robot able to achieve these tasks: to avoid obstacles and to make ones way toward its target. The algorithms are implemented in Matlab, afterwards tested with Matlab GUI; whereby the environment is studied in a two dimensional coordinate system. The simulation part is an approach to the real expected result; this part is done using Matlab to recognize all objects within the environment and since it is suitable for graphic problems. Taking the segmented environment issued from Matlab development, the algorithm permit the robot to move from the initial position to the desired position following an estimated trajectory using Maps in Matlab GUI.

\section{PROPOSED WORK}

There are two sections in project one is transmitter section and another is receiver section. Transmitter section consists of Matlab software, BLUETOOTH module where the receiver section consists of Arduino, BLUETOOTH module and Robot. The Robot mainly consists of two motors, Motor Driver IC L293D, Battery etc. As per the function of the robot The User once should have to give the path. User gives the required path through GUI (Matlab). So for this we made one pushbutton which is used for the drawing of map. Once user draw the map in GUI Window the IMDRAG function calculates the co-ordinates and gives a drag able path. Now the $\mathrm{X}$ and $\mathrm{Y}$ coordinates of the path is calculated and stored. Here we use simple Mathematical formula is used to find out the distance. So to find out we calculate distance between two coordinates Dx2-Dx1/Dy2-Dy1. So using this formula we get distance between all the coordinates of our path. To see what distance and angle we obtained from this map there are another pushbutton which shows all the parameters $n$ the command window of matlab.

Now to find out the angle we have to use the slope formula which is Tan-1 (Dx2-Dx1/Dy2-Dy1) where the Dx1 is the first co-ordinate and DX2 is second co-ordinate in X axis while DY1 is first co-ordinate and DY2 is second co-ordinate in $\mathrm{Y}$ axis. Now we have to store all this angle and distance in variables. According to this we get distance and angle now we have to decide the direction so for this just check out the angle what we get if it is NEGATIVE then turn Left and if POSITIVE then RIGHT and if it is 0 then go straight. Now these details are sending by transmitter section using BLUETOOTH Module. For this we use serial communication protocol. First, we have to initialize the serial communication for this we have to open the com port of the computer on which we connected the Arduino. For this we give one push button which open the comport, once the comport is open we can send the map to the Robot. For this we create a String which contains first distance, angle and direction.

Now the receiver receives the command from transmitter using the BLUETOOTH Module and given to the arduino. Arduino encodes all the String. Arduino Programming differentiates the Distance, Angle and Direction. All these parameters give the respective motion to The Robot. When the operation is done, we have to close the serial communication. To avoid accident the Ultrasonic sensor module is used which continuously check for obstacle and if found then take another respective action on it. So, for this it uses two pins Trigger and another is Echo the Trigger pin continuously transmit pulse. This Pulse is reflected back from the obstacle and the Echo pin receives it. The Time duration is calculated and corresponding to this time the Distance is calculated. This all makes the Robot reliable and efficient.

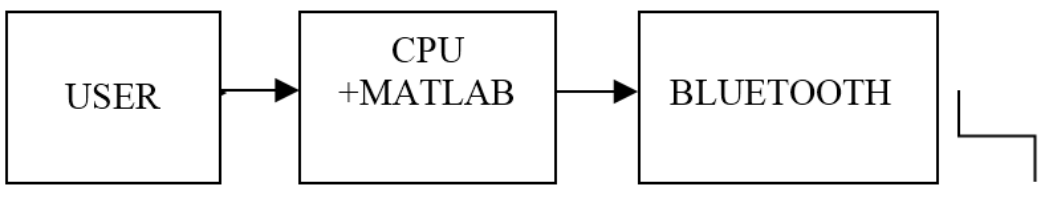

A) TRANSMITTER

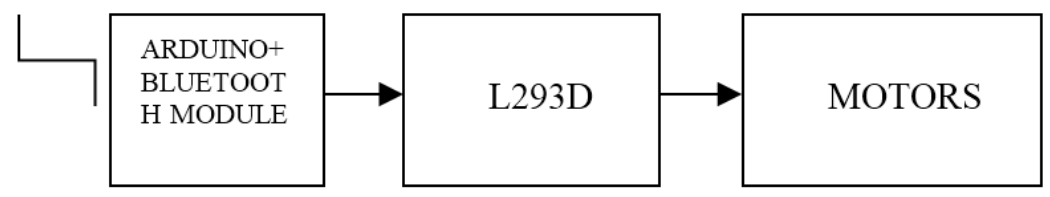

B) RECEIVER

Fig.1. Block diagram 
International Journal of Innovative Research in Electrical, Electronics, Instrumentation and Control Engineering

Vol. 8, Issue 8, August 2020

DOI 10.17148/IJIREEICE.2020.8801

Matlab Algorithm-

START

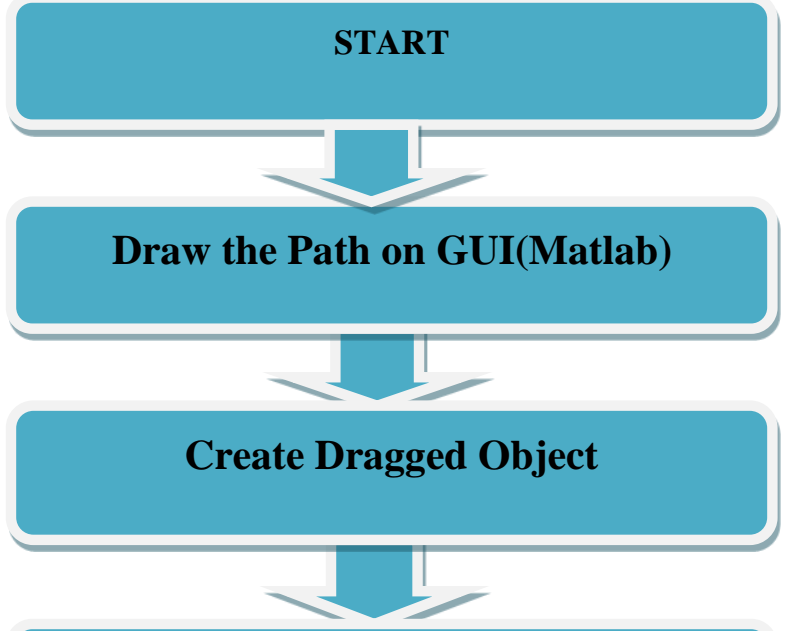

Get all the $X$ and $Y$ coordinates

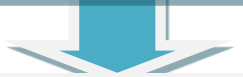

Find out Distance, Direction and Angle

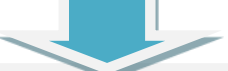

Send the String through Bluetooth

Arduino Algorithm-

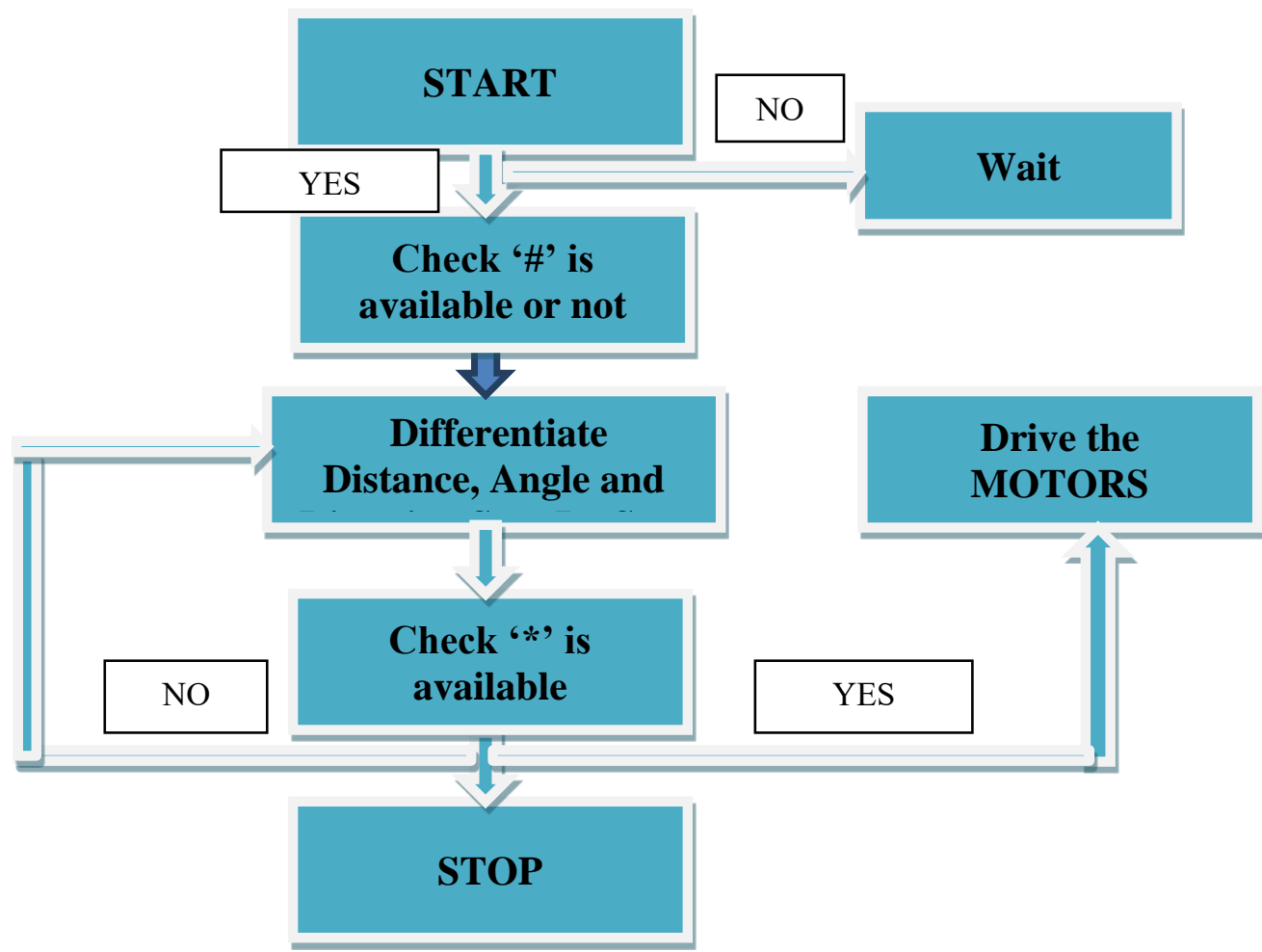


International Journal of Innovative Research in Electrical, Electronics, Instrumentation and Control Engineering

Vol. 8, Issue 8, August 2020

\section{DOI 10.17148/IJIREEICE.2020.8801}

\section{CONCLUSION}

We already know that The Path followed by Robot is Redesign able and also observed by user by GUI so it is User Convenient. Ultrasonic sensor informs about the obstacle appears in the path of Robot so its Accident Avoidable. Actual position of Robot can be monitored on the Screen. This robot is more reliable.

\section{REFERENCES}

[1]. “The WALK-MAN Robot in a Post earthquake Scenario",1070-9932/18@2018 IEEE.

[2]. "A LabVIEW-based Human-Computer Interaction System for the Exoskeleton Hand Rehabilitation Robot" proceeding of 2017, IEEE International Conference on Mechatronics and Automation. August 6-9, Japan

[3]. "Mapping and Pursuit-Evasion Strategies For a Simple Wall-Following Robot", Mapping and Pursuit-Evasion Strategies For a Simple WallFollowing Robot

[4]. "Mapping and Pursuit-Evasion Strategies For a Simple Wall-Following Robot", Mapping and Pursuit-Evasion Strategies For a Simple WallFollowing Robot

[5]. “A Map Building system for mobile Intelligent space using Human-Following Robot. 978-1-4799-0224-8/13/\$31.00 @2013 IEEE

[6]. "Past, Present and Future of Intelligent Robots", Proceedings 2003 IEEE International Symposium on Computational Intelligence in Rubotia and Automatiun July 16-20,2003. Kobe. Japan

\section{BIOGRAPHY}

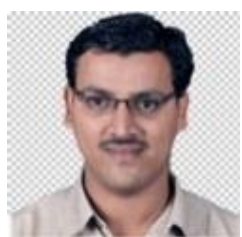

Mr. Prashant Maruti Jadhav working as an Assistant professor in Electrical engineering department of DKTE'S textile and engineering department. He completed master's degree in electronics engineering from Shivaji university, Kolhapur. He registered one patent. He is having academic experience of 9 years and ind ustrial experience of 4 years. 